deren Worten: höher als zuvor, aber noch relativ gering. Trotz des höheren Anteils von Frauen in Aufsichtsräten der allgemeinen Aktiengesellschaften ist der Anteil von Managerinnen heute nur sechs, fünf Prozent, nur zwei Prozent höher als in 2004.

In den Aufsichtsräten der privaten Aktiengesellschaften ist der Anteil von Frauen heute 17 Prozent, und dieser Anteil ist relativ stabil geblieben. Die gesetzlich regulierte Quote für beide Geschlechter in den Aufsichtsräten der allgemeinen Aktiengesellschaften scheint soweit keinen Effekt auf die privaten Aktiengesellschaften zu haben.

Im Allgemeinen haben sich die Karrieremöglichkeiten für Frauen parallel zur Entwicklung im Großteil der norwegischen Arbeitswelt geändert. Mit der Unterstützung eines starken gesetzlichen Hintergrundes, der die Diskriminierung auf der Grundlage unter anderem des Geschlechts verhindern soll, der flexiblen Regelung rund um Elternzeit und dem zunehmend guten Zugang zu Kindergärten und Kinderbetreuung haben Frauen wie Männer heute eine gute Möglichkeit, um die eigene Karriere mit einem fürsorglichen Familienleben zu kombinieren, was sich zweifelsohne positiv auf unsere Gesellschaft auswirkt.

Meine Damen und Herren, zwei zusammenfassende Punkte zum Schluss:

Die Firmen, die die Forderung nach Geschlechterrepräsentation erfüllt haben, haben erfahren, dass sie weder Ansehen, Umsatz oder Marktpositionen verloren haben. Die Forderung nach besserer Repräsentation der Geschlechter unterscheidet sich prinzipiell nicht von der Forderung nach der Anzahl der Aufsichtsratsmitglieder, der Angestelltenrepräsentation oder dem Wohnort der Aufsichtsratsmitglieder.

Wir sind in Norwegen einen Schritt weitergekommen, aber die Debatte über die Verbesserung der Repräsentanz der Frauen in der Wirtschaft ist noch nicht vorbei und wir erkennen, dass wir noch mehr zu tun haben.

In diesem Sinne wünsche ich allen einen interessanten und fruchtbaren Gedankenaustausch. Vielen Dank für Ihre Aufmerksamkeit!

\title{
Öffnet das System!
}

\section{Thomas Sattelberger \\ Vorstandsmitglied Deutsche Telekom AG, Bonn}

Exzellenz,

sehr verehrte Frau Pisal, meine Damen und Herren,

es ist mir Freude und Ehre zugleich, hier in der Königlichen Botschaft Norwegens zu Ihnen sprechen zu können. Norwegen ist bekanntermaßen Vorreiter in der Gleichstellung und Förderung von Frauen in Führungspositionen, nicht nur durch die Einführung der Frauenquote, sondern auch mit dem Weltfrauentag, der bereits seit 1913 in Norwegen begangen wird. Aber auch in Fragen der Work-Life-Balance ist Norwegen ganz vorne. Im OECD Better-Life Index belegt Norwegen den zweiten Platz, Deutschland den achten. Norwegen ist so ein Vorbild für Deutschland und viele andere europäische Länder. Die meisten von Ihnen wissen, dass FidAR und seine Präsidentin, Frau Schulz-Strelow, und ich, als Repräsentant der Deutschen Telekom, vor gut zwei Jahren am 15. März 2010 ein Fanal für Frauen in die Führung gesetzt haben, welches die gesellschaftliche und politische Debatte zum Thema befeuerte und den Jahrzehnte langsam kullernden Stein der Chancen-Fairness katalysatorisch beschleunigte. Die Zahl der Bekenntnisse und Appelle verhundertfachte sich - bei Freunden wie bei Gegnern. Doch der schnell rollende Stein lässt sich nicht mehr aufhalten.

Die Stunde der Wahrheit rückt näher: Zuerst bei den Aufsichtsräten:

Turnusmäßig stehen im Frühjahr 2013 eine ganze Reihe von Aufsichtsrats-Wahlen an. Somit beginnt nach der diesjährigen Hauptversammlungssaison die Uhr zu ticken.
Der Aufsichtsrat als oberstes Kontrollgremium eines Unternehmens prägt durch seine eigene Zusammensetzung die Unternehmenskultur mit. Gleichzeitig stellt ein klug zusammengesetzter Aufsichtsrat auch die Stellhebel zur Verbesserung der Aufstiegs- und Karrierechancen von weiblichen Führungskräften bis in den Vorstand. Es wird sich also in nächster Zukunft zeigen, ob der nötige Kulturwandel von den Aufsichtsrats-Vorsitzenden vorgelebt wird, oder ob wir in den vergangenen zwei Jahren nur Lippenbekenntnisse gehört haben. Von den 500 Aufsichtsratsmandaten der DAX30 sind in 2011 nur 80 auf beiden Aufsichtsratsbänken mit Frauen besetzt. Insgesamt beträgt der Anteil weiblicher Mandate - inklusive Arbeitnehmervertreterinnen - gerade einmal 16 Prozent, davon gerade einmal 29 dieser 80 auf Anteilseignerseite. Immer wieder höre ich das scheinheilige Argument, dass es nicht genug hoch qualifizierte Frauen gibt, die über Erfahrung und Expertise verfügen, um einen Aufsichtsratsposten in einem DAX-30-Konzern zu bekleiden. Dabei fehlen uns in der gesamten Bundesrepublik nur 70 weitere weibliche Aufsichtsratsmitglieder und wir hätten schon die 30-ProzentQuote erreicht. Ein hoch technologisiertes Land im Herzen Europas mit einer überdurchschnittlich gut ausgebildeten Bevölkerung von 82 Millionen Menschen will keine 70 qualifizierten Frauen aus seiner Mitte zusammenstellen können? Armes Deutschland!

Es gibt nur zwei Optionen: Nachdem Teile der CDU-Führung sich hinter der FDP verstecken, muss Frau Reding entweder vor den Wahlen die Quote durchsetzen oder zumindest das unabwendbare Damoklesschwert ziehen: „Wenn nicht... dann kommt sie!“ 
Im Europäischen Parlament ist die Mehrheit sicher. Die Stunde der Wahrheit kommt: Die Aufsichtsrats-Wahlen in 2013 müssen zu einem Quantensprung führen, sonst wird sich die Politik endlich ihrer Gestaltungsmacht bewusst - ansonsten ist sie wirklich Nachtwächter in dieser Frage.

Die Stunde der Wahrheit kommt auch auf dem zweiten Feld:

Die Zahl der weiblichen Vorstände erhöht sich nur im Schneckentempo, sozusagen auf der Kriechspur, noch dazu in deutlicher Schieflage.

Bis zum heutigen Zeitpunkt haben nur sieben DAX-Unternehmen Frauen in ihre Vorstände berufen. Nur acht Frauen - davon zwei von zehn Vorständen bei Siemens und bald zwei von sieben Vorständen bei Telekom - leiten die Geschicke der deutschen Top-Konzerne mit. Auch wenn weitere Berufungen bereits erfolgt sind - wie bei der Deutschen Post und jüngst auch bei BMW - steht das Männer-Frauen Verhältnis in Vorständen immer noch in einem grotesken Missverhältnis. Dazu kommt eine traurige Schieflage: Überwiegend bekleiden Frauen Vorstandstätigkeiten, die kein operatives Geschäft beinhalten. Von den aktuell acht und künftig elf weiblichen Vorstandsmitgliedern haben nur zwei eine Profit \& Loss-Verantwortung: Meine Kollegin Claudia Nemat, zuständig für den Vorstandsbereich Europa und damit für über 25 Prozent unseres Umsatzes sowie für den Technik-Bereich - ansonsten die Männerdomäne - sowie bei der Allianz, Helga Jung, verantwortlich für Iberia \& Lateinamerika. Die restlichen Frauen sind entweder reine Personal- oder Rechts-/Compliance-Vorstände oder verantworten im Kern diese Funktionen in Kombination mit zum Beispiel Personal, Einkauf, IT, Supply-Chain-Management oder Sustainability. Frauen sind nicht nur eine ganz rare Spezies im Vorstand, sondern bedienen das Klischee mit, dass Frauen eben gerade noch Personal und Administration, nicht aber operatives Geschäft „können“. Nachdem Frau Reding mit ihrer Quotendiskussion leider nur den Aufsichtsrat bedient und den Druck vom Vorstand nahm, kann man auf die Lage hier nur mit Zynismus oder Sarkasmus reagieren.

Bedrückend ist zudem, dass keine der Vorständinnen sich offensiv für eine Quote als ultima ratio aus dem Fenster gelehnt hat, obwohl fast alle von ihnen im Gefolge dieser Diskussion bestellt worden sind. Mut sieht anders aus! Von Januar 2011 bis Februar 2012 wurden insgesamt 27 Männer und sieben Frauen neu in den Vorstand eines deutschen DAX-30Konzerns berufen. Somit sind fast vier von fünf der berufenen Vorstände männlich - trotz des medialen, gesellschaftlichen und politischen Drucks, den Worten Taten folgen zu lassen. Und jetzt geht - jenseits von Personal und Administration die Luft raus. Die Blase platzt, wenn Frauen nicht für operatives Geschäft verantwortlich zeichnen dürfen.

Die Stunde der Wahrheit rückt auch bei den Ergebnissen der Selbstverpflichtung der DAX-30 näher.

Ich erinnere mich noch gut an das Treffen der DAX-Personalvorstände in Berlin. Als sich die DAX-Konzerne im März 2011die freiwillige Selbstverpflichtung auferlegt hatten, die Erhöhung des Frauenanteils in Führungspositionen - also für den Unterbau oder die Talentpipeline für Vorstand und Aufsichtsrat - zu garantieren, wollte man vor allem einer gesetzlichen Regelung zuvorkommen. Nicht viele hatten Herzblut zum Thema! Einige waren defensiv oder offen gegnerisch. Nichts desto weniger war der Schritt wichtig:

- Das Thema Frauen in Führung musste aus dem Hinterhof der betrieblichen Personalpolitik heraus. Wie erschrockene Rehe im Scheinwerferlicht standen viele DAX-30 zu diesem Thema auf der Straße der Öffentlichkeit.

- Zweitens wurde wohl erstmals in der Geschichte der DAX30 das Thema „Frauen in der Führung“ intensiv in vielen Vorstandsgremien überhaupt angesprochen.

- Drittens wird durch das öffentliche Nachhalten des Commitments dessen Ehrlichkeit sowie die Durchsetzungskraft des jeweiligen Personalverantwortlichen, des jeweiligen Gesamtvorstands, auf den Prüfstand gestellt

Hier bleibt die Bilanz abzuwarten, die wohl im Juni veröffentlicht wird. Sicher mit Licht und Schatten.

Wir als Deutsche Telekom haben vor über zwei Jahren, am 15. März 2010, unsere Haltung zum Thema „Frauen in der Führung " radikal reformiert:

Wir hatten uns im Vorstand damals aus eigenem Antrieb dafür entschieden, das Scheitern unserer traditionellen Wege der Frauenförderung einzugestehen und einen anderen, radikaleren Weg hinzuzufügen: nämlich als erstes DAX-30-Unternehmen eine - und ich werde dies belegen - machtvolle und kraftvolle Selbstverpflichtung einzugehen, bis Ende 2015 weltweit mindestens 30 Prozent des Managements - also 1200 der 4000 Führungspositionen - mit Frauen zu besetzen und mit dieser Verpflichtung an die Öffentlichkeit zu gehen.

Diese unsere Verpflichtung durchzieht den gesamten Konzern - angefangen an der Unternehmensspitze bis zur Rekrutierung von Nachwuchsinformatikerinnen. Ab dem 3. Mai 2012 wird mit meiner Nachfolgerin die Frauenquote im Konzernvorstand 29 Prozent betragen. Und im Aufsichtsrat beträgt unser Frauenanteil heute 20 Prozent. Bis 2013 ist es im Aufsichtsrat kein zu großer Sprung mehr, um auf die 30 Prozent zu gelangen.

Aber auch in den Führungsebenen zeigen sich die Früchte unserer Mühen. Von Oktober 2010 bis Ende 2011 hat sich unterhalb des Vorstands die Zahl der Frauen in Führungskräfte-Positionen signifikant verbessert: weltweit von 22,6 Prozent auf 24,7 Prozent und in unserer Telekom-Deutschland von 12,7 Prozent auf 15,6 Prozent.

Wenn meine Nachfolgerin, meine Vorstandskollegen und der Aufsichtsrat das Thema weiter mit Nachhaltigkeit, Sensibilität und hoher Projektmanagement-Disziplin begleiten, segelt unser Schiff bis 2015 in allen drei Dimensionen sicher in den 30-Prozent-Hafen und dann - mit kritischer Masse versehen - weiter nach vorne.

Ich sage das deshalb - auch Ihnen hier - mit allem Nachdruck, weil nur Kerzenlichter an der Tannenbaum-Spitze nicht nachhaltig sind. Symbolpolitik ohne Systematik bringt nichts.

Selbstverpflichtungen können beiderlei sein: gute moralische Geländer für Mächtige, die echte Veränderung wollen 
oder moralische Feigenblätter für Mächtige, die mit dem Erinnerungsverlust anderer spekulieren.

Eines möchte ich dem Juristinnenbund liebevoll ins Stammbuch mitgeben:

1. Prüfen Sie Selbstverpflichtungen ernsthaft auf Ehrlichkeit und wertschätzen Sie ehrliche Selbstverpflichtungen!

2. Setzen Sie nicht alleine auf die Symbolik der Tannenbaumspitze - wie gewonnen so zerronnen bei den geringen Zahlen -, sondern prüfen und fordern Sie, dass der gesamte „Tannenbaum der Personalentwicklung" leuchtet!

3. Bleiben Sie hartnäckigst dran: die Zeit spielt für Sie. Die Stunde der Wahrheit rückt immer näher!

4. Bauen Sie Koalitionen, auch und gerade mit Männervereinigungen oder männlich dominierten Vereinigungen!

5. Richten Sie Ihren Fokus nicht nur auf die börsennotierten Industrieunternehmen! Wichtige Gesellschaftsfelder müssen durchdrungen sein, zum Beispiel: Wissenschaft, Militär, Gesundheitswesen, Medien. Dort werden zentrale Prägungen vorgenommen.

- Nur in den Sprach- und Kulturwissenschaften liegt der Anteil der Professorinnen über 30 Prozent. In den Ingenieurwissenschaften beträgt ihr Anteil nur neun Prozent, im Bereich Mathematik/Naturwissenschaften zwölf Prozent.

- Die Bundeswehr scheitert weiterhin kläglich an der 15-Prozent-Quote (Gleichstellungsgesetz). Im Mai 2011 waren nur 9,3 Prozent der Bundeswehrsoldaten weiblich, davon 41 Prozent im Sanitätsdienst. Obwohl 65 Prozent der Soldatinnen Unteroffiziere sind, bleibt Führung im Militär Männerdomäne. Nur knapp 21 Prozent der Offiziere sind Frauen. Nur zwei Frauen wurden in der Geschichte der Bundeswehr jemals in den Generalsrang befördert - und auch nur als Generalärztinnen aus dem Sanitätsdienst. Eine davon ist seit 2004 bereits im Ruhestand. Bei den Spitzenverwendungen findet sich innerhalb der aktiven Streitkräfte weiterhin keine Frau.

- Frauenanteil Gesundheitswesen: Nur acht Prozent der Chefarztpositionen sind in Deutschland von Ärztinnen besetzt. Besonders zu adressieren ist die Frauenheilkunde, weil sie ein groteskes Beispiel für geschlechterspezifische Karriereverläufe ist. Der Anteil der Assistenzärztinnen in dieser Disziplin an den universitären Frauenkliniken beträgt 77 Prozent. Aber Führungsverantwortung bleibt selbst hier Männersache: In Deutschland gibt es nur zwei universitäre Chefärztinnen für Gynäkologie und Geburtshilfe an den mehr als 30 Universitätskliniken, somit sind 96 Prozent der universitären Klinikdirektionen in männlicher Hand! - abgesehen davon, dass die Frauen 20.000 Euro weniger verdienen als männliche Chefärzte.

- Auch der Frauenanteil bei Medienberufen ist erschreckend. Jüngst haben 350 Journalistinnen eine Frauenquote von mindestens 30 Prozent in der Führung in deutschen Medien gefordert. Das Schreiben an die deutschen Chefredakteure, Verleger und Intendanten nennt die Missstände beim Namen. Nur zwei Prozent aller deutschen Chefredakteure der rund 360 Tages- und Wochenzeitungen sind
Frauen. Von den 13 Intendanten des öffentlich-rechtlichen Rundfunks sind lediglich drei weiblich.

Ich habe bewusst Offiziere, Chefärzte, Lehrstuhlinhaber und Chefredakteure/Intendanten gewählt, weil sie - neben der Wirtschaft - das gesellschaftliche Problem voll ins Scheinwerferlicht setzen.

Der Weg in der Wirtschaft wird ein steiniger: Fast alle wichtigen politischen Veränderungsvorhaben für das System Arbeit - egal ob Vorstandsvergütungsgesetz 2009, Ausbildungspakt 2004 oder das Teilzeitarbeitsgesetz 2001 - sahen sich erbittertem Widerstand ausgesetzt, zum Teil sogar hoch ideologisiert. Von weiblicher Verschwörung ohne Qualität, über sozialistische An- und Eingriffe, bis hin zum Ende des freien Unternehmertums, der sozialen Marktwirtschaft oder gar dem Verfassungsbruch wird die Rede sein.

Gerade bei der Frauenquote wissen wir, dass wir es mit hartnäckigen Gegnerschaften zu tun haben. Tief verwurzelte, oft als selbstverständlich angesehene Gesellschaftsstrukturen, tradierte Stereotypen und Mentalitäten - sogenannte geschlossene Systeme - die mental wie faktisch geschlossen sind im Sinne von Verweigerung von Zugangsberechtigung sowohl für neue Ideen wie für andersartige Mitglieder.

Es bedarf eines radikalen Paradigmenwechsels. Wie Thomas Kuhn bereits feststellte, dauern Paradigmenwechsel oft jahrzehntelang. Die Worte von Max Planck „Wissenschaft entwickelt sich von Beerdigung zu Beerdigung weiter“ gelten auch für Kulturveränderung. Es bedarf der Beerdigung von festgefahrener Ideologie, Verhaltensweisen und mentalen Einstellungen, damit Neues entsteht. Denn die Geschichte lehrt uns: Geschlossene Systeme sind selten aus sich heraus reformierbar.

- In Anlehnung an die Suffragettenbewegung in England wurde 1904 in Berlin der „Weltbund für Frauenstimmrecht" gegründet mit dem Ziel, die Einführung des Frauenstimmrechts international zu koordinieren. Unter dem Druck des Ersten Weltkriegs forderte die bürgerliche und sozialdemokratische Frauenbewegung 1917 in gemeinsamer Resolution an den preußischen Landtag das aktive und passive Wahlrecht für Frauen. Die Durchsetzung erfolgte im November 1918.

- Die Rolle der Frau im wilhelminischen Kaiserreich: Die untergeordnete Rolle der Frau war fest in ihrer gesetzlich geregelten Beziehung zum Ehemann verankert. Der Mann verfügte in der Ehe über die rechtliche Erlaubnis, den Freiraum seiner Gattin einzuschränken. Diese Befugnis schloss auch das körperliche Züchtigungsrecht mit ein. Die Aufgaben der Frau waren im Preußischen Allgemeinen Landrecht geregelt.

- Die Rolle der Frau im dritten Reich: Die ideale Frau sollte sich neben der arischen Abstammung durch Treue, Pflichterfüllung, Opferbereitschaft, Leidensfähigkeit \& Selbstlosigkeit auszeichnen. Zum Wohle der „Volksgemeinschaft“ war die Pflichterfüllung als Mutter allem anderen vorangestellt. Zudem gab es nur ein sehr begrenztes Mitspracherecht in Ehe und Gesellschaft. 
- Die Maßnahmen durch das NS-Regime: Frauen wurden unter dem Vorwand des „Doppelverdienertums“ aus dem Arbeitsleben gedrängt. So wurden 1933 unter der Bedingung, dass die Ehefrau ihren Beruf aufgab, Ehestandsdarlehen an Ehemänner ausgezahlt. Die Abzahlung des Kredites verringerte sich jeweils um ein Viertel je Kindsgeburt.

- Frauen wurden beruflich heruntergestuft: im Schuldienst wurden Schulleiterinnen und Gymnasiallehrerinnen der höheren Schuljahre zunehmend durch männliche Lehrer ersetzt.

- Die Rolle der Frau im Nachkriegsdeutschland: Die Trümmerfrauen machten die Innenstädte bewohnbar und schafften die Grundlage für die Beseitigung von Kriegsschäden. Obwohl Frauen in den ersten Nachkriegsjahren in verschiedenen Wirtschaftsbereichen gearbeitet hatten, werden sie nach der Rückkehr der Männer aus Krieg und Kriegsgefangenschaft wieder nach Haus geschickt. Adenauers Frauen- und Familienideal sieht die Berufstätigkeit von Frauen nach der Eheschließung nicht vor. Auch in den Folgejahren war der Gesetzgeber in der Bundesrepublik nicht Antreiber beim Thema Frauenförderung.

- Ein Blick zurück verdeutlicht aber auch, dass alle Schritte der Emanzipation gesetzlich verankert wurden. Zur Erinnerung: Erst seit 1978 kann eine Frau bei der Eheschließung selbst ihren Namen bestimmen. Seit 1977 gibt es überhaupt ein souveränes Recht der Frauen auf Berufsausübung und es hat bis 1997 gedauert, dass Vergewaltigung in der Ehe unter Strafe gestellt wurde. Selbst bis 2001 war der „Dienst an der Waffe" für Frauen in der Bundeswehr undenkbar.

Die hart verdrahtete Quote war bei der Deutschen Telekom das Erfolgsrezept:

Unsere schmerzliche Einsicht bestand darin, uns einzugestehen, dass wir bis dahin nur die gleichen, tradierten Wege optimiert hatten: Das „Mehr des Gleichen“ als Lösung. Wir hatten nur im System gearbeitet, nicht am System:

- das X-te Frauen-Mentoring Programm, Patenschaftsmodelle, Cross Mentoring;

- das nächste Selbstmarketing-Training;

v das Versprechen, dass es „nächstes Jahr besser“ wird.

Erst mit dem Eingeständnis der Mächtigen, gescheitert zu sein und dem Unternehmermut der Mächtigen, unpopuläre Wege einzuschlagen, ließen sich jene Erfolge verzeichnen, die wir früher für unmöglich gehalten hatten.

Mächtige müssen mit Macht Fenster und Türen öffnen, sowie Treppen und Brücken bauen, um gläserne Decken und Wände zu beseitigen. Sie müssen das System öffnen für Diversity.

Der Paradigmenwechsel hin zur Gleichstellung der Frau und hin zu mehr Frauen in der Führung ist ein Ringen von Mächtigen, von moralischen Autoritäten verbunden mit wunderbaren Graswurzelbewegungen wie dem Deutschen Juristinnenbund.

Für Ihr Engagement und vor allem für Ihre Hartnäckigkeit danke ich Ihnen allen sehr. Ich bin an Ihrer Seite!

\section{Unconscious Bias}

\section{Dr. Brigitte Lammers}

Partnerin, Egon Zehnder International, Berlin

\section{Meine Damen und Herren,} zunächst bedanke ich mich sehr, heute bei Ihnen sein zu dürfen. Gestatten Sie mir auf Basis meiner Erfahrungen aus der Beratungs- und Besetzungspraxis dieser Tage eine bewusst andere Perspektive auf unseren Diskussionsgegenstand zu werfen. Wie Sie wissen, beschäftigt sich Egon Zehnder International unter anderem mit der Besetzung von Führungspositionen auf den Ebenen Vorstand, Geschäftsführung und Aufsichtsrat, ebenso wie auf anderen Leitungsebenen von Unternehmen. Werfen wir also einen Blick auf die positiven wie negativen Entwicklungen in solchen Besetzungsprozessen und lassen Sie uns diskutieren, welche Schlussfolgerungen daraus abgeleitet werden können. Erlauben Sie mir, Ihnen diese Überlegungen in zehn kurzen Thesen vorzustellen:

These 1: Über die Notwendigkeit der Verstärkung der Aufsichtsrats- und Vorstandsebene, ebenso wie anderer Leitungsebenen, mit weiblichen Kandidatinnen wird in unseren Klientenkreisen nicht mehr grundsätzlich diskutiert. Eine an sich banale Feststellung, die aber einen großen Fortschritt markiert. In den letzten zwei bis drei Jahren war das deutlich anders. Während wir vor wenigen Jahren durchaus noch erleben konnten, dass Unternehmen sich bewusst gegen die Einstellung von weiblichen Führungskräften aussprachen, weil die „Kultur“ des jeweiligen Unternehmens dies vermeintlich noch nicht zuließ, so hat sich dies in nahezu allen Unternehmensumfeldern und -größen radikal geändert. Über das „Ob“ der Einstellung und Förderung weiblicher Führungskräfte wird heute nicht mehr gesprochen, über das „Wie“ wird demgegenüber nach wie vor durchaus heftig gestritten.

These 2: Wir alle verfolgen mit Blick auf jüngste Presseveröffentlichungen, dass die deutschen Aufsichtsräte weiblicher werden. Dies erfolgt allerdings im Rahmen des derzeit „Möglichen“, denn Vakanzen sind zurzeit noch rar. Die große Neubesetzungswelle - wir haben es heute schon einige Male gehört - steht erst für das Jahr 2013 bevor. Dennoch sehen wir in diesen Tagen bei den auftretenden Vakanzen, dass sich Unternehmen ganz mehrheitlich, insbesondere im börsennotierten Umfeld, in Richtung weiblicher Kandidatinnen orientieren. Die in einer durchaus bemerkenswerten fachlichen Breite vorgenommenen Besetzungen der letzten Monate entziehen bereits heute dem Argument die Grundlage, dass es nicht ge- 